\title{
IL VENTO SULL'ALTIPIANO CARSICO DI TRIESTE
}

\author{
Silvio Polli
}

1. Premessa. - La citta di Trieste, il suo golfo e il retrostante sollevamento carsico sono soggetti, per la loro particolare posizione, ad uno speciale e caratteristico regime di venti. Nel semestre estivo, con condizioni meteoriche normali, si hanno regolari brezze di mare e di terra. Nel semestre invernale queste sono sopraffatte, quasi in continua alternanza, dal violento e secco vento da ENE (bora) e dai moderati e umidi venti da SE (scirocco). 11 Carso, che ad un'altezza di circa $300 \mathrm{n}$ si leva immediatamente a $\mathrm{N}$ e a $\mathrm{NE}$ della città, più che ostacolare la discesa della bora, la favorisce, sia come soglia aperta sul mare, sia come effettiva circostanza concomitante alla sua generazione. La bora si presenta con la massima intensità nei mesi invernali, ma non è rara, meno violenta perì, anche nei mesi estivi. Si precipita dall'altipiano mantenendo direzione molto costante. Assume massima violenza lungo i rarchi aventi la medesima direzione. Pur riscaldandosi nella caduta per compressione adiabatica, rimane sempre un vento freddo e secco.

Lo studio della bora era stato eseguito sulla base delle registrazioni anemometriche eseguite a Trieste e delle osservazioni stimate eseguite sul Carso. Queste ultime, fatte senza l'aiuto di strumenti, si limitano alle tre osservazioni griornaliere delle ore 7,14 e 19. Hanno valore puramente indicativo basandosi esse sul presupposto che la bora sull'altipliano fosse più intensa che sulla citti. Si presentava perciò sempre di grande interesse la sistemazione di anemografi sull'altipiano carsico e sul suo margine. Ciò, per varie circostanze, non è stato possibile realizzare prima di questi ultimi anni. Appena alla fine del 1947, il locale Lfficio Agricoltura del Governo Militare Alleato, venuto a conoscenza della questione, dispose subito perché fossero messi alcuni anemometri sull'altipiano e nei dintorni di Trieste. L'ideale sarebbe stato potere disporre di apparechi registratori della direzione, della velociti media e delle raffiche. La spesa risultava troppo forte e perciò furono messi in funzione dei semplici anemometri a coppe, totalizzatori, a lettura diretta.

Presentiamo in questa nota $i$ risultati di due delle cinque sta- 
zioni attualmente in funzione. Essi rappresentano le prime misure anemometriche eseguite sul Carso. I valori ottenuti sono rigorosamente confrontabili fra di loro e con quelli di ogni altro anemografo. I risultati sono pertanto di notevole importanza sia scientifica che pratica. In un certo senso essi sono anche sorprendenti. Si riteneva infatti che la bora fosse sull'altipliano più forte che in città, ciò che invece non risultò dall'esame delle serie di misure eseguite. La questione era dovuta puramente a impressioni psicologiche. Indubhiamente, per quanto si è detto precedentemente, la hora è sul Carso più fredda che al mare. Inoltre, la mancanza di ripari, il suolo con copertura invernale spesso gelata, l'implacalile costanza della direzione che il paesaggio nudo non riesce ad alterare, sono tutte circostanze che fanno apparire, a chi generalmente vive nella citta in siti riparati, più forte di quanto in realtà non lo sia la velocita del vento sul Carso.

Dai dati che si presentano in questa nota si deduce invece che la velocità media della hora è i $3 / 4$ di quella di Trieste a Basovizza, e i $2 / 3$ a Villa Opicina. Inoltre, da sopraluoghi eseguiti in periodi di hora in varie zone dell'altipiano, risultò pure che le raffiche sono meno marcate che in città. La hora sul Carso ha caratteristiche di maggriore uniformità. La minore velocità e soprattutto la ridotta vorticosità lasciano intravedere la possibilità di sistemare sull'altopiano degli efficienti aeroporti, la cui costruzione risulta invece impos.shile in città.

2. La stazione di Opicina. -- $\mathbf{\mathrm { E }}$ situata a SE del pacse, in posizione leggermente elevata rispetto al nucleo centrale della località, nella villa a due piani di Via del Ricreatorio n. 16. Lo strumento è sistemato sul terrazzino terminale dell'edificio. Le coppe sono a $2,5 \mathrm{~m}$ sopra di esso e a $17 \mathrm{~m}$ sul suolo. Le coordinate geografiche della stazione sono: lat. $45^{\circ} 41^{\prime} 09^{\prime \prime} \mathrm{N}$, long. $13^{\circ} 47^{\prime} 22^{\prime \prime} \mathrm{E}$ Gr., $1^{\circ} 20^{\prime} 14^{\prime \prime} \mathrm{E}$ M. Mario (Roma), altezza del suolo m 330 sul livello medio marino.

L'apparecchio risulta in posizione dominante il paese ed $\grave{e}$ sufficientemente libero a tutti i venti. Il sollevamento marginale carsico, che si eleva $60 \mathrm{~m}$ più alto a circa $800 \mathrm{~m}$ verso $\mathrm{SW}$, ripara alquanto tutta la località dai venti da Sud e da SW.

Lo strumento è un anemometro totalizzatore Salmoiraghi a 3 coppe. La distanza dell'asse di rotazione dal centro delle coppe è di cm 17, il diametro delle coppe è di $\mathrm{cm} 8$. Esso indica il numero dei metri percorsi dal vento. Le letture si eseguono giornalmente alle 
Tabella I - Velocità media del vento a Opicina.

\begin{tabular}{|c|c|c|c|c|c|c|c|c|c|c|c|c|c|}
\hline Anno & I & II & III & IV & v & $V_{1}$ & VII & V'III & IX & $\mathrm{x}$ & XI & XII & Anno \\
\hline 1948 & 5, & 11,2 & 9,6 & 9,8 & 7,8 & 6,9 & 7,9 & 7,4 & 6,7 & 10.1 & 11.9 & 13.6 & 9.0 \\
\hline 1949 & 10.8 & 9.7 & 144 & 6.4 & 8.3 & 7,9 & 59 & 83 & 71 & 132 & 125 & 76 & 9.3 \\
\hline 1950 & 14.7 & 8.1 & 10.7 & 8.1 & 8.5 & 7.5 & 83 & 6,4 & 69 & 99 & 7.4 & 10.4 & 89 \\
\hline 1951 & 4.5 & 120 & 10.6 & 9.7 & 6.6 & 6.9 & 7.4 & 6.7 & 91 & 17.1 & 7.2 & 6.1 & 9.1 \\
\hline 1952 & 7.4 & 7.0 & 7.4 & 8.0 & 6.2 & 7.1 & 8.3 & 54 & $(1.9$ & 6.7 & 5.9 & 15.4 & 76 \\
\hline Media & 9.6 & 97 & 10,5 & 8.4 & 7.5 & 7.3 & 7.6 & 68 & 7.3 & 11.4 & 9.0 & 10.6 & 8.8 \\
\hline
\end{tabular}

Tabella II - Velocità media del vento nei singoli quadranti.

\begin{tabular}{|c|c|c|c|c|c|c|c|c|c|c|c|c|c|}
\hline Anno & I & II & 111 & IV & $v$ & VI & VIII & VIII & $1 \mathrm{x}$ & $\mathrm{x}$ & XI & XII & A nno \\
\hline $\begin{array}{l}{ }^{\circ} Q . \\
19^{8} 8\end{array}$ & 7.1 & 129 & 11.8 & 10.9 & 100 & 7.6 & 9.6 & 12.1 & 83 & 126 & 13.0 & 15.3 & 10.9 \\
\hline 1949 & 127 & 13.2 & 15.7 & 9.8 & 136 & 10.8 & 77 & 11.1 & 10.5 & 156 & 161 & 9.1 & 12.2 \\
\hline 1950 & 18.9 & 10.8 & 152 & 92 & 13.0 & 10.8 & 98 & 8.4 & 8.1 & $17 \cdot 2$ & 109 & 12.3 & 12.1 \\
\hline 1951 & 16.8 & 126 & 148 & 17. & 8.1 & 10.1 & 9.6 & 9.1 & 12.7 & 19.1 & 9.3 & 103 & 12.5 \\
\hline 1952 & 10.0 & 119 & 11.6 & 12.5 & 96 & 76 & 9.7 & 6.7 & 9.2 & 8.7 & 8.7 & 20.4 & 10.6 \\
\hline Medin & 13.1 & 12.3 & 13.8 & 12.0 & 109 & 9.1 & 9.3 & 9.5 & 9.8 & 14.6 & 11.6 & 13.5 & 11.7 \\
\hline 1948 & 65 & 2.8 & 67 & 8.0 & 6.8 & 74 & 8.1 & 55 & 5.1 & 5,0 & 6.2 & 54 & 6.1 \\
\hline 1949 & 11.3 & 55 & 0.0 & 72 & 43 & 5.1 & 5.2 & 9.4 & 4.2 & 63 & 8.1 & 5.8 & 6.0 \\
\hline 1950 & 32 & 7.8 & 4.9 & 6.8 & 7.0 & 7.1 & 0.0 & 5.4 & 5.6 & 5.1 & 65 & 6.4 & 5.5 \\
\hline 1951 & 5.9 & 118 & 83 & 6.0 & 5.7 & 46 & 5.9 & 5.2 & 5.2 & 39 & 6.7 & 41 & 6.1 \\
\hline 1952 & 42 & 4.4 & 3.1 & 6.8 & 5.5 & 6.5 & 7.6 & 5.8 & 61 & 55 & +3 & 7.4 & 5.6 \\
\hline $\begin{array}{l}\text { Medin } \\
\text { LIOOO. }\end{array}$ & 6.2 & 6.5 & 4.6 & 7.0 & 5.9 & 6.1 & 5.4 & 6.3 & 5.2 & 5,2 & 6.4 & 5.8 & 5,9 \\
\hline 1948 & 1.8 & 2.7 & 00 & 0.0 & 00 & 98 & 00 & 5.9 & 4.1 & 0.0 & 0.0 & 4.0 & 2.4 \\
\hline 19.19 & 1.6 & 0.0 & 0.0 & 6.7 & 4.9 & 00 & 49 & 1.0 & 0.0 & 8.9 & 7.2 & 2.6 & 31 \\
\hline 1950 & 1.9 & $\begin{array}{ll}0 & 0\end{array}$ & 00 & 91 & 94 & 6.8 & 1.8 & 4.8 & 0.0 & 2.8 & 0.0 & $\begin{array}{ll}00 \\
0\end{array}$ & 3.2 \\
\hline 1951 & 3.1 & 35 & 6.5 & 50 & 49 & 5.8 & +18 & 5.5 & 61 & 00 & 26 & 3.9 & 4.3 \\
\hline 1952 & 0.0 & 28 & 30 & 39 & 42 & 7.0 & 8.1 & 4.4 & 4.7 & 6.9 & $1 . t$ & 45 & 4.2 \\
\hline $\begin{array}{l}\text { Media } \\
\text { lvogy }\end{array}$ & $1 . \bar{\imath}$ & 1.8 & 1.9 & 4.9 & +.7 & 5.9 & 4.5 & 4.1 & $\therefore 0$ & 3.7 & 2.2 & 3.0 & 3.4 \\
\hline 1918 & 2.3 & 2.6 & f. & 5.3 & 50 & ร. 2 & 3.8 & 4.5 & 4.3 & 2.9 & 0.0 & 0.0 & 3.5 \\
\hline 1949 & 4.9 & 3.4 & 54 & 48 & +6 & 4.5 & 4.5 & 4.7 & 4.1 & 4.3 & 6.7 & 2.0 & 45 \\
\hline 1950 & 9.3 & 4.8 & 4.4 & 5.3 & 5.0 & 42 & 68 & 4.2 & 3.4 & 00 & 3.0 & 00 & 4.2 \\
\hline 1951 & 22 & 00 & 5.7 & 6.8 & 45 & 0.0 & 00 & 66 & 0.0 & 0.0 & 0.0 & 5.7 & 2.6 \\
\hline 195: & 0.0 & 47 & 27 & 25 & 40 & 7.5 & 5.4 & 46 & 44 & 3.8 & 3.2 & 0.0 & 3.6 \\
\hline Media & 3.7 & 3.1 & 1.9 & 4.9 & 4.6 & 43 & 4.1 & 4.9 & 3.2 & 2,2 & 2.6 & 1.5 & 3.7 \\
\hline
\end{tabular}

TaBElla III - Velocità media della bora.

\begin{tabular}{|c|c|c|c|c|c|c|c|c|c|c|c|c|c|}
\hline Anno & I & II & III & IV & V & VI & VII & Vill & IX & $\mathrm{x}$ & $\mathbf{X I}$ & XII & Anno \\
\hline 1948 & 9.4 & $1 i .2$ & 121 & 11.4 & 112 & 7.7 & 101 & 18.0 & 9.7 & 18.1 & 16.1 & 186 & 133 \\
\hline 1949 & 134 & 14.8 & 177 & 10,3 & 149 & 11.2 & 8,2 & 12.1 & 11.9 & 17.9 & 20.0 & 13.4 & \\
\hline 1950 & 263 & 11.7 & 10.3 & 11.1 & 13.0 & 12.4 & 10.1 & 8.4 & 8.5 & 20.4 & 120 & 147 & 13.7 \\
\hline 1951 & 19.0 & 15.8 & 16.2 & 16.4 & $\begin{array}{l}10,8 \\
\end{array}$ & 11.5 & 10.1 & 9.3 & 15.0 & 208 & 12.0 & 108 & 14.1 \\
\hline 1952 & 10.9 & 13.7 & 14.4 & 15.8 & 12.5 & 7.8 & 10.2 & 7.8 & 9.7 & 9.9 & 10,2 & 22.6 & 12.1 \\
\hline Media & 15.8 & 14.6 & 153 & 13.2 & 12.5 & 10.1 & 9.7 & $11: 1$ & 11.0 & 174 & 14.1 & 16.0 & 13.4 \\
\hline
\end{tabular}


ore 8. Osservatore è il maestro Giovanni Pauli che esegue pure le altre osservazioni meteoriche.

3. La stazione di Basovizza. - E situata a SE del paese, al n. 109, presso la casa forestale della Provincia. Il sito è pianeggiante e aperto a tutti i venti. Il margine carsico, situato $1500 \mathrm{~m}$ verso $\mathrm{SE}$, è di pochi metri più elevato del paese e pertanto influisce poco, quale ostacolo, sul regime locale del vento. Le coordinate geografiche della stazione sono: lat. $45^{\circ} 38^{\prime} 30^{\prime \prime} \mathrm{N}$, long. $13^{\circ} 51^{\prime} 33^{\prime \prime} \mathrm{E} \mathrm{Gr}$. 1 $1^{\circ} 24^{\prime} 25^{\prime \prime} \mathrm{E}$ M. Mario (Roma), la quota al suolo è di $375 \mathrm{~m}$ sul livello medio marino.

Lo strumento è fissato alla sommita di un palo con le coppe a m 6,05 dal suolo. E un anemometro Salmoiraghi totalizzatore a 3 coppe di alluminio. La distanza dall'asse di rolazione al centro delle coppe è di cm 17, il diametro delle coppe è di $\mathrm{cm}$ 8. Le letture sono eseguite ogni giorno alle ore 8 .

4. La stazione di Trieste. - Lo strumento è sistemato sulla torre dell'Istituto Talassografico, situato nella parte SIV della città, al n. 2 del Viale Romolo Gessi. La posizione è bene aperta ai venti del I, III e IV quadrante, è invece leggermente riparata dai venti del II quadrante dagli alti edifici costruiti negli ultimi anni sul promontorio di S. Andrea. Le coordinate geografiche della torre sono: lat. $45^{\circ} 38^{\prime} 37^{\prime \prime} \mathrm{N}$, long. $13^{\circ} 45^{\prime} 09^{\prime \prime} \mathrm{E} \mathrm{Gr}$, $1^{\circ} 18^{\prime} 03^{\prime \prime} \mathrm{E}$ M. Mario (Roma). Il mulinello a coppe è fissato all'estremità di un tubo d'acciaio, $7 \mathrm{~m}$ sopra la terrazza terminale, a $38 \mathrm{~m}$ dal suolo e a $45 \mathrm{~m}$ sul livello medio del mare.

Lo strumento è un anemografo Universale S.I.A.P. a 3 coppe e con il tubo di Pitot sull'asse della banderuola, contro vento. Esso registra con continuita i $\mathrm{km}$ percorsi, la direzione e le raffiche del vento. Il raggio dall'asse di rotazione al centro delle coppe è di cm. 21,8, il diametro esterno delle coppe è di $\mathrm{cm} \mathrm{16,0.} \mathrm{Lo} \mathrm{scorrimento}$ della carta del diagramma è di 22,5 mm per 1 ora.

5. Elaborazione delle misure. - Dalle letture eseguite alle ore 8 si dedussero le velocità medie gionaliere corrispondenti alle 24 ore precedenti. Dalle medie giornaliere si ottennero poi quelle mensili. Queste pertanto si riferiscono al vento percorso dalle ore 8 del giorno 1 di ciascun mese alle ore 8 del griorno 1 del mese seguente.

Anche i dati di Trieste corrispondono arrli stessi intervalli di 
Tabelua IV - Velocità media del vento a Basovizza.

\begin{tabular}{|c|c|c|c|c|c|c|c|c|c|c|c|c|c|}
\hline Anno & I & II & III & IV & $\mathrm{v}$ & vI & Vil & VIII & IX & $\mathrm{x}$ & XI & XII & Anno \\
\hline 1941 & $\begin{array}{ll}51 \\
1\end{array}$ & 12.4 & 9.5 & 118 & 7.7 & 83 & 7.7 & 7.2 & 6.3 & 11.7 & 12.7 & 14.2 & 9.6 \\
\hline 1949 & 106 & 9.3 & 15.1 & 92 & 8.6 & 8.9 & 6.0 & 8.3 & 68 & 14.3 & 13.3 & 7.3 & 98 \\
\hline 1950 & 136 & 89 & 10.2 & 10.2 & 9.6 & 7.4 & 79 & 61 & 6.8 & 12.2 & 9.9 & 12.8 & 9.6 \\
\hline 1951 & 10.3 & 12.3 & 11.1 & 125 & 7.1 & 6.5 & 7.3 & 6.6 & 9.6 & 19.7 & 85 & 5.6 & 9.8 \\
\hline 1952 & 8.2 & 8.6 & 62 & 80 & 6.0 & 6.9 & 8.0 & 4.5 & 6.3 & 6.6 & 5.1 & 16.6 & 7.6 \\
\hline Medin & 96 & 10.3 & 104 & 10.3 & 78 & 7.6 & 74 & 6.5 & 7.2 & 12.9 & 9.9 & 11.3 & 93 \\
\hline
\end{tabular}

Tabella V -- Velocità media del vento nei singoli quadranti.

\begin{tabular}{|c|c|c|c|c|c|c|c|c|c|c|c|c|c|}
\hline Inno & I & II & III & IV & $v$ & II & VII & VIII & IX & $x$ & XI & XII & Anno \\
\hline $\begin{array}{l}100 . \\
1999\end{array}$ & 6.5 & $1+1.2$ & 11.3 & 13.6 & 11.6 & 7,8 & 105 & 11.8 & 7.6 & 14.1 & 14.0 & 15.7 & 11.6 \\
\hline 1949 & 12.0 & 13.5 & 16.4 & 15.4 & 144 & 12.5 & 8.2 & 6.3 & 9.7 & 17.0 & 16.8 & 8.7 & 126 \\
\hline 1950 & 17.3 & 11.7 & 14.6 & 11.3 & 16.8 & 10.8 & 9.8 & 8.3 & 7.9 & 21.6 & 17.1 & 13.8 & 13.4 \\
\hline 191 & 16.8 & 13.8 & 14.8 & 94.8 & 9.0 & 9.8 & 9.9 & 8.9 & 13.0 & 22.1 & 9.4 & 9.4 & 13.5 \\
\hline 1952 & 10.9 & 15.4 & 9.1 & 10.1 & 9.4 & 7.5 & 9.7 & 61 & 8.1 & 8.9 & 7.3 & 21.6 & 10.3 \\
\hline Mediu & 12.7 & 13.7 & 13.2 & 15.0 & 12.2 & 9.7 & 9.6 & 8.3 & 9.3 & 16.7 & 12.9 & 13.8 & 12.3 \\
\hline $\begin{array}{l}1100 . \\
1948\end{array}$ & 69 & 2.7 & 6.9 & 96 & 6.7 & 8.8 & 8.1 & 5.6 & 4.7 & 4.4 & 59 & 5.4 & 6.3 \\
\hline 1949 & 10.2 & 4.9 & 0.0 & 7.3 & 41 & 5.6 & 7.6 & 11.1 & 4.5 & โ.3 & 90 & 5.7 & 6.5 \\
\hline 19.20 & 35 & 8.1 & 5.2 & 9.9 & 6.1 & 85 & 0.0 & 7.1 & 6.3 & 64 & 7.8 & 10.6 & 6.6 \\
\hline 1951 & 7.0 & 11.0 & 9.1 & 0.5 & 6.3 & +8 & 5.6 & 5.0 & 6.2 & 4.2 & 8.7 & 3.9 & 6.5 \\
\hline 1952 & 4.9 & 5.4 & 3.7 & 8.7 & 58 & 60 & 7.6 & 50 & 6.0 & 5.6 & 42 & 9.4 & 6.1 \\
\hline Medin & 6.5 & 6.4 & 5.0 & 8.4 & 58 & 6.9 & 5.8 & 6.8 & 5.5 & 4.7 & 7.1 & 7.0 & 6.4 \\
\hline 1948 & 3.1 & 2.7 & $\begin{array}{lll}0 & 0\end{array}$ & 0.0 & $\begin{array}{ll}0 & 0\end{array}$ & 10.7 & 0.0 & 5.6 & 3.0 & $\begin{array}{ll}0 & 0\end{array}$ & 0.0 & 2.7 & 23 \\
\hline 1949 & 1.8 & 0.0 & 0.0 & 7.0 & 4.4 & 0.0 & 5.0 & 0.0 & 00 & 8.9 & 8.1 & 2.6 & 3.2 \\
\hline 1950 & 2.6 & 0.0 & 0.0 & 11.4 & 10.8 & 6.4 & 3.9 & 4.0 & 0.0 & 3.1 & 0.0 & 0.0 & 3.5 \\
\hline 19.51 & 4.0 & 4.0 & 8.2 & 48 & 5.0 & 4.3 & +2 & 5.4 & 6.1 & 0.0 & 2.5 & 3.4 & 4.3 \\
\hline 1952 & 0.0 & 3.2 & 2.5 & 5.1 & 2.7 & 5.8 & 7.0 & 3.5 & 4.3 & 5.5 & 0.9 & 5.1 & 3.8 \\
\hline Medin & 2.3 & 2.0 & 2.1 & 5.7 & 46 & 5.4 & 4.0 & 3.7 & 2.7 & 35 & 23 & 2.8 & 3.4 \\
\hline $\begin{array}{l}1500 . \\
1918\end{array}$ & 3.7 & 2.3 & 6.9 & 6.1 & 5.1 & 4,5 & 28 & 3.5 & 3.2 & 2.6 & 0.0 & 0.0 & 34 \\
\hline 1949 & 5.8 & 2.7 & 6.4 & 6.8 & 4.1 & 4.4 & 4.0 & 3.5 & 4.0 & 2.9 & 7.2 & 32 & 4.6 \\
\hline 1950 & 8.9 & 5.0 & 3.5 & 5.7 & 4.5 & 3.8 & 5.9 & 3.4 & 2.7 & 0.0 & 5.9 & 0.0 & 4.1 \\
\hline 1951 & 7.1 & 0.0 & 6.1 & 8.1 & 7.3 & 0.0 & 0.0 & 7.1 & 0.0 & 00 & 0.0 & 6.3 & 3.5 \\
\hline 1952 & 0.0 & 3.4 & 3.7 & 2.0 & 3.6 & 6.3 & 5.0 & 3.1 & 3.9 & 3.8 & 1.6 & 0.0 & 3.0 \\
\hline Medin & 5.1 & 2.7 & 5.3 & 5.7 & 5.0 & 3.8 & 3.5 & 4.1 & 2.8 & 19 & 2.9 & 19 & 3.7 \\
\hline
\end{tabular}

Tabela VI - Velocità media della bora.

\begin{tabular}{|c|c|c|c|c|c|c|c|c|c|c|c|c|c|}
\hline Inno & 1 & II & III & IV & $v$ & $v_{1}$ & vil & VIII & IX & $x$ & XI & NII & Anno \\
\hline 19. & 9.1 & 19.4 & 11.3 & 144 & 13.1 & 8.3 & 11.1 & 20.5 & 9.6 & 2) 8 & 16.8 & 19.5 & 14.5 \\
\hline 19 & 14.7 & 15.9 & 18.2 & 6.7 & 16.0 & 13.3 & 8.6 & 12.9 & 11.5 & 181 & 20.5 & & 9 \\
\hline 1950 & 24.0 & 128 & 15.7 & 13.4 & 16.8 & 12.4 & 9.6 & 8.3 & 8.6 & 26.0 & 13.4 & $1+9$ & 14.7 \\
\hline 1951 & 19.. & 17.6 & 16.4 & 24.8 & 12.3 & 114 & 10,4 & $6 . t$ & 14.8 & $\because 4.2$ & 12.4 & 9.8 & 15.0 \\
\hline 1952 & 11.4 & 18.2 & 10.3 & 13.0 & 10.0 & 7.9 & 10.1 & 7.8 & 8.8 & 9.9 & 8.0 & 231 & 11.5 \\
\hline Media & 157 & 16.8 & 14.4 & 165 & 13.6 & 10.7 & 10.0 & 112 & 8.9 & 198 & 14.2 & 15.9 & 14.0 \\
\hline
\end{tabular}


tempo e pertanto i valori delle tre stazioni sono, da questo punto di vista, rigorosamente confrontabili.

Le tabelle 1,4 e 7 presentano i valori medi mensili e annui delle velocità, espresse in $\mathrm{km} /$ ora, per ciascuna delle tre località. Sono cioè i quozienti fra il numero dei $\mathrm{km}$ percorsi da tutti i venti nelle singole stazioni ed il numero delle ore contenute nel mese.

I venti, secondo le loro direzioni di provenienza, furono aggruppati in quadranti secondo lo schema seguente:

I Quadrante, comprende i venti da NNE ad E,

II Quadrante, comprende i venti da ESE a $\mathrm{S}$,

III Quadrante, comprende i venti da SSW a W,

IV Quadrante, comprende i venti da WNW a $\mathrm{N}$.

Le tabelle 2, 5 e 8 danno le velocità medie in $\mathrm{km} /$ ora corrispondenti ai venti avuti secondo i quadranti ora definiti.

Particolare importanza assume la conoscenza del comportamento della bora sull'altipiano in confronto a quello che essa manifesta a Trieste e sul golfo. La sua individuazione ̀̀ molto facilitata dal fatto che essa proviene da ENE e mantiene molto costante la sua direzione. Sul diagramma essa figura perciò ben determinata. È pertanto possibile ricavare da questo dati molto precisi sulla sua velocita e frequenza.

Nelle tabelle 3,6 e 9 sono date in $\mathrm{km} /$ ora, le velociti medie del vento da ENE, cioè della bora. Esse sono i quozienti fra il numero di $\mathrm{km}$ percorsi dalla bora in ciascun mese ed il corrispondente numero di ore durante le quali essa ha soffiato.

Nelle ultime tabelle $(10,11$ e 12$)$ sono riportate, per ogni localíà, le medie quinquennali e ciò per avere la possibilità di cseguire un immediato confronto tra i valori delle tre stazioni.

6. Considerazioni conclusive. - a) Sull'altipiano carsico la velocità media del vento risulta minore che a Trieste e nella zona del porto. A Basovizza essa ̀̀ il $78 \%$ di quella registrata a Trieste, a Opicina il $74 \%$. A determinare tale fatto concorrono tutti i principali venti della regione: la bora, lo scirocco e le brezze. Si vedrà più avanti perché questi venti sono meno forti sul Carso che a Trieste.

b) Per la bora, ciò̀ il vento da ENE, questi risultati sono ancora più marcati. I corrispondenti valori percentuali sono per Basovizza $71 \%$ e per Opicina $68 \%$. Nella prima località la velocità della bora è circa i 3/4 di quella di Trieste, nella seconda circa i $2 / 3$. Ciò è dovuto in 
Tabella VII - Velocità media del vento a Trieste.

\begin{tabular}{|c|c|c|c|c|c|c|c|c|c|c|c|c|c|}
\hline Anno & 1 & 11 & III & IV & v & vI & vil & vill & IX & $\mathrm{x}$ & XI & XII & Anno \\
\hline 1948 & 6.0 & 15.0 & 12.3 & 12.6 & 9.1 & 9.5 & 11.7 & 10.3 & 85 & 16.0 & 15.3 & 17.0 & 11.9 \\
\hline 1949 & 12.8 & 10.9 & 18.3 & 6.7 & 126 & 10.7 & 7.6 & 11.6 & 9.6 & 20.5 & 19.0 & 9.0 & 12.4 \\
\hline 1950 & 21.3 & 9.1 & 13.4 & 94 & 10.8 & 10.1 & 11.7 & 8.0 & 9.5 & 14.8 & 10.1 & 15.1 & 11.9 \\
\hline 1951 & 13.1 & 14.3 & 14.1 & 12.9 & 10.4 & 9.4 & 10.5 & 9.8 & 12,7 & 22.9 & 101 & 8.0 & 12,4 \\
\hline 1952 & 10.9 & 11.0 & 9.6 & 11.2 & 9.3 & 10.2 & 112 & 8.1 & 11.2 & 10.7 & 8.7 & 21.4 & 11.1 \\
\hline Media & 12.8 & 12.1 & 13.5 & 10.6 & 10.4 & 10.0 & 10.5 & 96 & 10.3 & 17.0 & 12,6 & 142 & 11.9 \\
\hline
\end{tabular}

TABELLA VIII - Velocità media del vento nei singoli quadranti

\begin{tabular}{|c|c|c|c|c|c|c|c|c|c|c|c|c|c|}
\hline Anno & 1 & 11 & III & IV & v & VI & VII & vill & $1 X$ & $\mathbf{x}$ & XI & XII & Anno \\
\hline 100. & & & & & & & & & & & & & \\
\hline 19.48 & 7.6 & 17.7 & 16.1 & 14.2 & 12.6 & 11.2 & 16.2 & 19.8 & 12.4 & 22.4 & 17.6 & 19.9 & 15.6 \\
\hline $\begin{array}{l}19 .+9 \\
10-0\end{array}$ & 18.3 & 10.4 & 211.2 & 13.3 & 24.2 & 16.9 & 11.2 & 18.4 & 16.8 & 25.5 & 27.9 & 11.9 & 18.1 \\
\hline 1951 & 0.3 & 10.4 & 20.0 & 10.9 & 20.3 & 17.1 & 15.7 & 12.2 & 12.7 & 26.5 & 15.3 & 17.4 & 17.8 \\
\hline 1952 & 13.8 &. .4 & 19.0 & 17.0 & $\begin{array}{l}14.9 \\
12.5\end{array}$ & 12.0 & 13.9 & 12.0 & 17.5 & 2.5 & 12.8 & 15.0 & .2 \\
\hline & & & & & & & & & & & & 20.1 & 10.2 \\
\hline $\begin{array}{l}\text { motna } \\
11^{\circ}(1) .\end{array}$ & 18.1 & 17.2 & 18.2 & 15.9 & 16.9 & 13.8 & 14.2 & 14.5 & 14.8 & 22.7 & 17.1 & 18.5 & 16.8 \\
\hline 1948 & 6.7 & 1.7 & 6.9 & 11.0 & 7.4 & 11.4 & 7.9 & 6.8 & 5.2 & 2.7 & 3.9 & 1.7 & 6.1 \\
\hline 1949 & $\overline{7.3}$ & 3.7 & 0.0 & 6.8 & $\because 2$ & 4.8 & 7.5 & 15.0 & 2.9 & 8.0 & 7.0 & 4.4 & 5.9 \\
\hline 1950 & 1.9 & 5.6 & 3.7 & 8.9 & $\therefore 4$ & 7.8 & 0.0 & 6.0 & 4.9 & 7.6 & 8.8 & 11.2 & 5.9 \\
\hline 1951 & .9 & 13.6 & 10.9 & 7.6 & 7.8 & $\because .2$ & 7.9 & 8.1 & 7.8 & 4.9 & 9.6 & 5.5 & 4.2 \\
\hline 1952 & 7.3 & 6.6 & 4.4 & 10.3 & 9.0 & 9.5 & 8.0 & 9.6 & 10.1 & 8.9 & 7.0 & 10.8 & 8.5 \\
\hline Medial & 6.2 & 6.2 & 5.2 & 8.9 & 6.0 & 8.1 & 6.3 & 9.1 & 6.2 & 6.4 & 7.3 & 6.5 & 6.9 \\
\hline 19.18 & 2.1 & 3.6 & $\left(1,{ }^{\prime}\right)$ & 0.1 & 0.0 & 16.3 & 0.0 & $\tau .9$ & 1.4 & 0.0 & 0.0 & 0.4 & 2.6 \\
\hline 19,9 & 1.5 & 0.0 & 0.0 & 5.7 & 4.0 & 0.0 & 3.7 & 0.0 & 0.0 & 6.0 & 7.4 & 0.8 & 2.4 \\
\hline 1950 & 2.3 & 0.0 & 0.0 & 9.6 & 11.9 & 7.8 & 3.1 & 5.7 & 0.0 & 4.7 & 0.0 & 0.0 & 3.8 \\
\hline 1951 & 4.8 & 6.0 & 9.9 & 6.7 & 6.9 & 8.4 & 6.8 & 8.6 & 8.1 & 0.0 & 2.5 & 5.3 & 6.2 \\
\hline 1952 & 0.0 & 3.4 & 3.9 & 5.3 & 7.0 & 8.11 & 11.3 & 6.5 & 8.6 & 12.8 & 2.4 & 1.0 & 6.3 \\
\hline $\begin{array}{l}\text { Mrdia } \\
1100 .\end{array}$ & 2.1 & 2.6 & 2.8 & 5.5 & 6.0 & 8.1 & 5.0 & 5.7 & 3.6 & 4.7 & 2.5 & 2.6 & 4.3 \\
\hline 1918 & 1.6 & 1.5 & 8.1 & 5.2 & 4.9 & 4.9 & 2.7 & 4.2 & 2.0 & 2.3 & 0.0 & 0.0 & 3.1 \\
\hline 1919 & 3.7 & 1.3 & 5.8 & 3.6 & 4.3 & 3.2 & 5.0 & 3.0 & 3.4 & 2.0 & 9.9 & 1.0 & 3.9 \\
\hline 1951 & 10.5 & 1.7 & 3.2 & 3.3 & 4.2 & 3.5 & 7.4 & 3.1 & 2.5 & 0.0 & 3.1 & 0.0 & 3.6 \\
\hline 1911 & 2.6 & 0.0 & 6.0 & 8.7 & 7.5 & 0.0 & 0.0 & 10.5 & 0.0 & 0.0 & 0.0 & 9.0 & 3. \\
\hline 1952 & 0.11 & 3.7 & 4.9 & 3.6 & 5.8 & 11.1 & 7.3 & 5.6 & 6.5 & 4.5 & 5.1 & 0.0 & 4.8 \\
\hline Media & 3.7 & 1.6 & 5.6 & 4.9 & 5.3 & 4.5 & 4.5 & 5.3 & 2.9 & 1.8 & 3.6 & 2.0 & 3.8 \\
\hline
\end{tabular}

Tabelia IX - Velocità media della bora.

\begin{tabular}{|c|c|c|c|c|c|c|c|c|c|c|c|c|c|}
\hline Anno & 1 & II & III & IV & v & $\mathrm{VI}_{\mathrm{I}}$ & v1I & vill & $1 \mathrm{x}$ & $\mathrm{x}$ & XI & xll & Amo \\
\hline 1918 & 11.5 & .8 & 16 & 15.4 & 15.4 & $115^{-}$ & $1: 8$ & 32.5 & 15.5 & 31.0 & 22.5 & 25.4 & 20.3 \\
\hline 1949 & 19.3 & i 9.3 & 22.9 & 14.3 & 2.8 & 17.6 & 12.0 & 20.7 & 20.0 & 29.3 & 36.3 & 18.9 & 21.5 \\
\hline 1950 & 40.6 & 17.9 & 22.8 & 14.6 & 20.3 & 20.5 & 15.9 & 12.2 & 13.3 & 32.1 & 15.3 & 20.8 & 20.5 \\
\hline 1451 & 20.6 & 19.4 & 22,1 & 23.6 & 18.1 & 11.9 & 14.7 & 11.7 & 20.5 & 27.9 & 15.5 & 15.8 & 19.2 \\
\hline 1952 & i 5.6 & 23.5 & It. 3 & 21.9 & 15.8 & 11.8 & 14.8 & 11.9 & 15.3 & 15.7 & 13.4 & 31.8 & 17.5 \\
\hline Media & $: 2.7$ & 21.0 & 20.5 & 18.0 & 19.5 & 15.3 & 15.0 & 17.8 & 16.9 & 27.9 & 20.6 & 22.5 & 19.8 \\
\hline
\end{tabular}


TABELLA X - Velocità media del vento (1948-52).

\begin{tabular}{|l|r|r|r|r|r|r|r|r|r|r|r|r|r|}
\hline Lociliti & $\mathrm{I}$ & $\mathrm{II}$ & $\mathrm{III}$ & $\mathrm{r}$ & $\mathrm{v}$ & $\mathrm{vI}$ & $\mathrm{vII}$ & $\mathrm{vII}$ & $\mathrm{Ix}$ & $\mathrm{x}$ & $\mathrm{xI}$ & $\mathrm{xII}$ & Anno \\
\hline Opicina & 9.6 & 9.7 & 105 & 8.4 & 7.5 & 7.3 & 7.6 & 6.8 & 7.3 & 11.4 & 9.0 & 10.6 & 8.8 \\
Basovizza & 9.6 & 10.3 & 10.4 & 10.3 & 7.8 & 7.6 & 7.4 & 6.5 & 7.2 & 12.9 & 9.9 & 11.3 & 9.3 \\
Trieste & 12.8 & 12.1 & 13.5 & 10.6 & 10.4 & 10.0 & 10.5 & 9.6 & 10.3 & 17.0 & 12.6 & 14.2 & 11.9 \\
\hline
\end{tabular}

TAB. XI - Velocità media del vento nei singoli quadranti (1948-52).

\begin{tabular}{|c|c|c|c|c|c|c|c|c|c|c|c|c|c|}
\hline Lo.a.tlà & 1 & II & III & IV & $\mathbf{v}$ & VI & VII & VIII & $1 x$ & $x$ & XI & XII & Anno \\
\hline óuladr. & & & & & & & & & & & & & \\
\hline $\begin{array}{l}\text { Opicina } \\
\text { Basovizza }\end{array}$ & $\begin{array}{l}13.1 \\
12.7\end{array}$ & $\begin{array}{l}12.3 \\
13.7\end{array}$ & $\begin{array}{l}13.8 \\
13.2\end{array}$ & $\begin{array}{l}12.0 \\
15.0\end{array}$ & $\begin{array}{l}10.9 \\
12.2\end{array}$ & $\begin{array}{l}9.4 \\
9.7\end{array}$ & $\begin{array}{l}9.3 \\
9.6\end{array}$ & $\begin{array}{l}9.5 \\
8.3\end{array}$ & $\begin{array}{l}9.8 \\
9.3\end{array}$ & $\begin{array}{l}11.6 \\
16.7\end{array}$ & $\begin{array}{l}11.6 \\
12.9\end{array}$ & $\begin{array}{l}13.5 \\
13.8\end{array}$ & $\begin{array}{l}11.7 \\
12.3\end{array}$ \\
\hline Iriesle & 18.1 & 17.2 & 18.2 & 15.9 & 16.9 & 13.8 & 14.2 & 14.5 & 14.8 & 22.7 & 17.1 & 18.5 & 16.8 \\
\hline$\|^{\circ}$ Quadr. & & & & & & & & & & & & & \\
\hline Opicuna & 6.2 & 6.5 & 4.6 & 7.0 & 5.9 & 6.1 & 5.4 & 6.3 & 5.2 & 5.2 & 6.4 & 5.8 & 5.9 \\
\hline Basovizza & 6.5 & 6.4 & 5.0 & 8.4 & 5.8 & 6.9 & 5.8 & 6.8 & 5.5 & 4.7 & 7.1 & 7.0 & 6.4 \\
\hline Trieste & 6.2 & 6.2 & 5.2 & 8.9 & 6.6 & 8.1 & 6.3 & 9.1 & 6.2 & 6.4 & 7.3 & 6.5 & 6.9 \\
\hline Illoquadr. & & & & & & & & & & & & & \\
\hline Upicina & 1.7 & 1.8 & 1.9 & 4.9 & 4.7 & 5.9 & 4.5 & 4.1 & 3.0 & 3.7 & 2.2 & 3.0 & 3.4 \\
\hline Basovizza & 2.3 & 2.0 & 2.1 & 5.7 & 4.6 & 5.4 & 4.13 & 3.7 & 2.7 & 3.5 & 2.3 & 2.8 & 3.4 \\
\hline Trieste & 2.1 & 2.6 & 2.8 & 5.5 & 6.0 & 8.1 & 5.0 & 5.7 & 3.6 & 4.7 & 2.5 & 2.6 & 4.3 \\
\hline WVo@uadr. & & & & & & & & & & & & & \\
\hline Upicina & 3.7 & 3.1 & 4.9 & 4.9 & 4.6 & 4.3 & 4.1 & 4.9 & 3.2 & 2.2 & 2.6 & -1.5 & 3.7 \\
\hline Basovizza & 5.1 & 2.7 & 5.3 & 5.7 & 5.0 & 3.8 & 3.5 & 4.1 & 2.8 & 1.9 & 2.9 & 1.9 & 3.7 \\
\hline |r.esle & 3.7 & 1.6 & 5.6 & 4.9 & 5.3 & 4.5 & 4.5 & 5.3 & 2.9 & 1.8 & 3.6 & 2.0 & 3.8 \\
\hline
\end{tabular}

TABELLA XII - Velocità media della bora (1948-52).

\begin{tabular}{|c|c|c|c|c|c|c|c|c|c|c|c|c|c|}
\hline Località & 1 & II & III & IV & $\mathbf{v}$ & $v_{1}$ & VII & VIII & IX & $\mathrm{x}$ & XI & XII & Anno \\
\hline Opicl & 15.8 & 14.6 & 15.3 & 13.2 & 12.5 & 10.1 & 9.7 & 11.1 & 11.0 & 17.4 & 14.1 & 16.0 & 13.4 \\
\hline Basovizza & 15.7 & 16.8 & 14.4 & 16.5 & 13.6 & $1_{0.7}$ & 10.0 & 11.2 & 8.9 & 19.8 & 14.2 & 15.9 & 14.0 \\
\hline Trieste & 22.7 & 21.0 & 20,5 & 18.0 & 19.5 & 15.3 & 15.0 & 17.8 & 16.9 & 27.9 & 20.6 & 22.5 & 19.8 \\
\hline
\end{tabular}

parte al fatto che sull'altipiano il moto delle masse d'aria avviene su una zona estesa, secondo dislivelli e gradienti, sia termici che barometrici, discreti; in parte all'essere la bora un vento freddo di caduta che precipitando dal margine dell'altipiano al mare aumenta gradatamente la sua velocità. Ne consegue che sulla città e sul porto la bora assume caratteri di vorticosità che sull'altipiano difficilmente possono realizzarsi. Per l'insieme dei venti del I quadrante valgono valori percentuali poco diversi, dato che la bora ne è il vento predominante fra essi.

c) Per i venti del II quadrante, che comprendono quelli da scirocco, si hanno pure velocità medie minori sull'altipiano rispetto alla 
città e al mare. A Opicina e Basovizza essi risultano rispettivamente l'85 e il 93\% di quelli registrati a Trieste. I venti sciroccali, provenienti dal mare, colpiscono direttamente la città e si smorzano lentamente nel sollevarsi sull'altopiano e nell'allontanarsi dal mare.

d) Ciò vale pure per i venti del III quadrante, che comprendono quelli da libeccio e le brezze di mare e di terra. Queste ultime, allontanandosi dalla zona costiera, si indeboliscono rapidamente. Dato il predominio delle brezze, si ha che $\mathrm{i}$ valori massimi delle velocità carlono nei mesi estivi, i minimi in quelli invernali.

e) I venti del IV quadrante comprendono quelli del Nord, che provenendo dal retroterra scendono al mare dopo aver sorpassato le due località carsiche. Le velocità medie figurano ancora lievemente inferiori sull'altipiano. La discesa lungo i pendii marginali fa acquistare a questi venti freddi una maggiore velocità, per cui arrivano sulla città con una velocità incrementata del $3 \%$ circa.

f) Tra le due località carsiche considerate risulta sempre più ventosa quella di Basovizza. Ciò dipende dal fatto che essa si trova ad una quota maggiore e in una zona leggermente più esposta. Opicina invece giace in posizione leggermente concava e pertanto più riparata.

Trieste - Istituto Talassografico - Gennaio 1953.

\section{RIASSUNTO}

Si presentano $i$ risultati di cinque anni di rilievi anemometrici eseguiti nelle località di Opicina e Basovizza, sull'altipiano carsico di Trieste. Si confrontano $i$ dati con quelli di Trieste mettendo in particolare evidenza il regime della "bora". Risulta che questa, contrariamente all'opinione generale, $\grave{e}$ meno intensa e meno vorticosa sul Carso che al mare.

\section{SUMMARY}

The results of five years of anemometric reliefs carried out in Opicina and Basovizza (Carsic tableland of Trieste) are presented here. These data are confronted with the data of Trieste, making especially evidente the regimen of the "bora". The result is that this, on the contrary of the general opinion, is less intens and less whirling on the Carso that on the sea. 


\section{BIBLIOGRAFIA}

S. Pollı, 100 anni di osservazioni meteorologiche eseguite a Trieste. Parte IV (La velocità del vento), P. V. (La direzione del vento), P. VI (Le raffiche del vento); Boll. Società Adriatica di Scienze Naturali, Trieste, vol. XLIV (1948), XLV (1949), XLVI (1950).

S. Polli, Le correnti aeree al suolo e in quota a Trieste. Riv. di Meteorologia Aeronautica, a. IV, n. 2, Roma 1940.

S. Poll., Dati climatici di Trieste e dintorni. Pubbl. n. 284, Istit. Talassogr., Trieste 1952. 